\title{
Thank you to our reviewers 2014
}

\author{
Abdur Rehman, Karim \\ Abrams, David \\ Agaku, Israel \\ Aghi, Mira \\ Al-alawy, Khamis \\ Al-hamdani, Mohammed \\ Allem, Jon-Patrick \\ Allen, Sharon \\ Allwright, Shane \\ Amos, Amanda \\ Anderson, Stacey \\ Arnott, Deborah \\ Arora, Monika \\ Ayo-Yusuf, Olalekan \\ Babb, Stephen \\ Bala, M \\ Balbach, Edith \\ Bansal-Travers, Maansi \\ Barber, Sarah \\ Barnes, Richard \\ Barnoya, Joaquin \\ Beaudoin, Chris \\ Benowitz, Neal \\ Berteletti, Florence \\ Bialous, Stella \\ Bittencourt, Lorna \\ Blanco Marquizo, Adriana \\ Blank, Melissa \\ Blecher, Evan \\ Bloch, Michele \\ Bogdanovica, Ilze \\ Bohac, David \\ Boman-Davis, Marie \\ Bonevski, Billie \\ Borland, Ron \\ Branston, J. \\ Brennan, Emily \\ Breysse, Patrick \\ Britton, John \\ Brose, Leonie \\ Brown, Cati \\ Brown, Jamie \\ Brown, Jennifer \\ Bucolo, Sebastiano \\ Buettner-Schmidt, Kelly \\ Bullen, Chris \\ Burns, David \\ Burton, Suzan \\ Callard, Cynthia \\ Camenga, Deepa \\ Cantrell, Jennifer \\ Cappella, Joseph \\ Caraballo, Ralph \\ Carter, Owen \\ Cavazos-Rehg, Patricia \\ Chaloupka, Frank \\ Chapman, Simon \\ Chelwa, Grieve \\ Cheng, Kai-Wen \\ Cherukupalli, Rajeev
}

Choi, Kelvin

Chopra, Amandeep

Clegg Smith, Katherine

Collin, Jeff

Conway, Kevin

Cornelius, Monica

Costafreda, Sergi

cox, alison

Crane, Julian

Cruz, Tess

Cui, Kainan

Cummings, Michael

Cummins, Sharon

Curry, Laurel

Daher, Nancy

Daube, Michael

de Andrade, Marisa

de Beyer, Joy

de Moura, Lenildo

Delnevo, Cristine

Dewhirst, Timothy

Diethelm, Pascal

Ding, Shirley

Dobbins, Tim

Donny, Eric

Douglas, Cliff

Drope, Jacqui

Drope, Jeffrey

Dubray, Jolene

Duffy, Sheila

Dunlop, Sally

Efroymson, Debra

Eissenberg, Thomas

El Sabban, Marwan

Elton-Marshall, Tara

Emily, Falk

Emory, Kristen

Evans, Sarah

Farrelly, Matthew

Fathelrahman, Ahmed

Fellows, Jeffrey

Ferrence, Roberta

Ferris Wayne, Geoffrey

Fleischer, Nancy

Ford, Allison

Forsyth, Susan

Foulds, Jonathan

Francis, John

Fraser, Doug

Freeman, Becky

Freiberg, Mike

Gallopel-Morvan, Karine

Gallus, Silvano

Gan, Quan

Ganz, Ollie

Gao, Junling

Gartner, Coral

Gendall, Philip

Gilmore, Anna

Giovino, Gary
Glantz, Stanton

Glynn, Thomas

Goniewicz, Maciej

Gorini, Giuseppe

Gottlieb, Mark

Gourdet, Camille

Grana, Rachel

Greenland, Steven

Greenwold, Mark

Guindon, Emmanuel

Gupta, Prakash

Guydish, Joseph

Haddock, Christopher

Hahn, Ellen

Haifley, Gregg

Hammond, David

Hanewinkel, Reiner

Harrington, Kathleen

harris, fiona

Hastings, Gerard

Hatsukami, Dorothy

Havard, Alys

Hawkins, Benjamin

Haydon, Colleen

Hearn, Bryan

Hickman, Norval

Hiscock, Rosemary

Hitchman, Sara

Hoek, Janet

Hornsby, Paige

Hovell, Melbourne

$\mathrm{Hu}$, Teh-wei

Huang, Jidong

Hurt, Richard

Jaine, Richard

Jawad, Mohammed

Jha, Prabhat

Jo, Catherine

John, Rijo

Jones, Miranda

Joossens, Luk

Joossens, Luk

Kaleta, Dorota

Kasza, Karin

Kegler, Michelle

Kenkel, Donald

Kennedy, Ryan David

Khan, Javaid

Khang, Young-Ho

Kilgore, Elizabeth

Kim, Annice

King, Bill

King, Brian

Kirchner, Thomas

Klein, Elizabeth

Kornfield, Rachel

Kostova, Deliana

Krawitz, Marilyn

Kunst, Anton

Lai, Foon Yin
Lam, Tai Hing

Langley, Tessa

Leatherdale, Scott

Lee, Seowting

Lee, Youn

LeGresley, Eric

levy, david

Levy, Douglas

Lewis, Germaine

Lewis, M. Jane

Lewis, Sarah

Liber, Alex

Liberman, Jonathan

Licht, Andrea

Lightwood, James

Lim, Min Kyung

Lin, Tsai-yu

Ling, Pamela

Lipkus, Isaac

Liu, Ruiling

Liu, Sherry

López-Nicolás, Ángel

Luke, Douglas

Lund, Ingeborg

Mackay, Judith

MacKillop, James

MacKintosh, Anne-Marie

Maloney, Erin

Mannocci, Alice

Maubach, Ninya

Max, Wendy

Maynard, Olivia

Mays, Darren

Maziak, Wasim

McCool, Judith

McDaniel, Patricia

McGrady, Benn

McKee, Martin

Mead, Erin

Merriman, David

Messer, Karen

Moeller-Saxone, Kristen

Mons, Ute

Moodie, Crawford

Moran, Meghan

Morris, Daniel

Muggli, Monique

Mukherjea, Arnab

Munafo, Marcus R.

Murukutla, Nandita

Muttappallymyalil, Jayakumary

Nagelhout, Gera

Nair, Urmila

Nakkash, Rima

Navas-Acien, Ana

Nesson, Erik

Niederdeppe, Jeff

Nigar, Nargis

Nonnemaker, James 
OConnor, Richard

Onder, Zeynep

Ong, Michael

Ort, Christoph

Palipudi, Krishna

Pankow, James

Pappas, R

Pearson, Jennifer

Peeters, Silvy

Pepper, Jessica

Peppone, Luke

Pergadia, Michele

Peruga, Armando

Plotnikova, Evgeniya

Pokhrel, Pallav

Popova, Lucy

Pratt, Angela

Prochaska, Judith

Ramos Carbajales, Alejandro

Raw, Martin

Rees, Vaughan

Reid, Jessica

Ribisl, Kurt

Rice, Jerry

Rogers, Todd

Romer, Dan

Rooke, Catriona
Ross, Hana

Rossi, Jean-Pierre

Rousu, Matthew

Rutkow, Lainie

Saebo, Gunnar

Saenz de Miera Juarez, Belen

Samet, Jonathan

Sanders-Jackson, Ashley

Sangthong, Rassamee

Savelli, Mat

Scheffels, Janne

Schensul, Jean

Schroeder, Steve

Schroth, Kevin

Scollo, Michelle

Semple, Sean

Shafey, Omar

Shaffer, Ellen

Shah, Sarwat

Shang, Ce

Sharma, Priyamvada

Shastri, Aditi

Shiffman, Saul

Siahpush, Mohammad

Singh, Rana

Smith, Andrea

Smith, Elizabeth
Smith, Philip

Soni, Preeti

Southwell, Brian

Stellman, Steven

Stepanov, Irina

Stillman, Frances

Stoklosa, Michal

Sullivan, Ryan

Sung, Hai-Yen

Sutfin, Erin

Sweeting, Helen

Tait, Peter

Talley, Brandon

Tan, Andy

Thomas, RE

Thompson, Francis

Thomson, George

Thornley, Simon

Thrasher, James

Toll, Benjamin

Travers, Mark

Vallone, Donna

van der Deen, Frederieke

Van Walbeek, Corne

Vellios, Nicole

Villanti, Andrea

Volchan, Elaine
Voon, Tania

Wackowski, Olivia

Wakefield, Melanie

Walker, N

Wang, Man Ping

Ward, Kenneth

Warner, Kenneth

Wazir, Muhammad

West, Oliver

West, Robert

White, Jason

Whyte, Gregor

Widome, Rachel

Wipfli, Heather

Woodward, Alistair

$\mathrm{Xu}, \mathrm{Xin}$

Yang, Gong

Yao, Tingting

Yong, Hua

Young, David

Young-Wolff, Kelly

Yurekli, Ayda

Zawertailo, Laurie

Zhang, Bo

Zheng, Rong 